\title{
Predicting the extent of dissolution of a cloud of particles entering a liquid.
}

\author{
J. W. Holbeach M. R. Davidson*
}

(Received 8 August 2003)

\begin{abstract}
Dissolution and dispersion of solids in liquids is important in areas as diverse as extractive metallurgy and the dairy industry. A numerical model is developed to examine the effect of dissolution upon the dispersion of solids entering an initially quiescent bath. Computations are based upon a pseudo two dimensional finite volume, Eulerian-Eulerian, multiphase model. Dissolution is shown to significantly affect dispersion of solids with solid liquid density ratios greater than one. A dimensionless group equal to the ratio of the initial particle terminal velocity to the dissolution velocity is shown to be useful in predicting the extent of dissolution at specific penetration depths.
\end{abstract}

*Department Chemical and Biomolecular Engineering, University of Melbourne Australia. mailto: j.holbeach@pgrad.unimelb.edu.au

See http://anziamj.austms.org.au/V45/CTAC2003/Holb for this article, (c) Austral. Mathematical Soc. 2004. Published July 6, 2004. ISSN 1446-8735 


\section{Contents}

1 Introduction

C605

2 Formulation

C606

2.1 Model development . . . . . . . . . . . . . . . . C608

2.2 Dissolution . . . . . . . . . . . . . . . . . . . . C609

3 Discussion

C611

4 Conclusion

C615

References

C615

\section{Introduction}

Dissolution and dispersion of solids entering a liquid is important in areas as diverse as extractive metallurgy, the dairy industry, and mineral processing. The process may be as common as salt dissolving in water or, in an alloying process, molybdenum particles dissolving in molten steel. Examples involving addition to metallurgical melts include: pelletized directly reduced iron added to an electric arc furnace [1], or the addition of alloying materials such as nickel or aluminium.

In previous work, a computational model successfully and accurately predicted the dispersion of inert solid particles in liquids. Suitable techniques developed in the context of Nuclear reactor safety [2, 3, 4] were extended by Smith [5] for the dispersion of a solids stream entering an initially quiescent liquid.

Dissolution is a well understood process. Numerical and experimental models developed in the metallurgical arena cover specific cases. For example, nickel is often alloyed with zinc for galvanizing of steel. Langberg and 
Nilmani [6] developed a model of the dissolution of nickel into zinc. This model, based upon work by Apelian et al. [7], was simplified to allow an analytical rather than numerical solution.

While the above are just a few examples of modelling of solid particle dispersion and dissolution, there is little modelling work of these two effects in conjunction. The inclusion of dissolution alters the dispersion of solids in various ways. A changing particle diameter will alter the drag force experienced and so can have a significant effect upon the penetration and dispersion. Mass transfer may lead to changes in the liquid density, momentum transfer from the solid to the liquid phase, or liquid viscosity changes due to additional components being dissolved into the liquid, all of which may be important. For example, in the case of salt dissolution into water, density variations normally result in natural convection [8]. We have previously [9] discussed the effect of dissolution upon particles dropped into an initially quiescent liquid. This paper extends that work to present and discuss the dependence of penetration depth on a dimensionless group which is the ratio of the initial particle terminal velocity to particle dissolution velocity.

\section{Formulation}

The present work examines the penetration of a short pulse of dissolving spherical solid particles (of initial diameter $6.25 \mathrm{~mm}$ ) dropped into an initially quiescent fluid. The pulse or cloud of particles dissolves and disperses as it descends. The computational simulation is based on the model of Smith [13] for non-dissolving particles. 


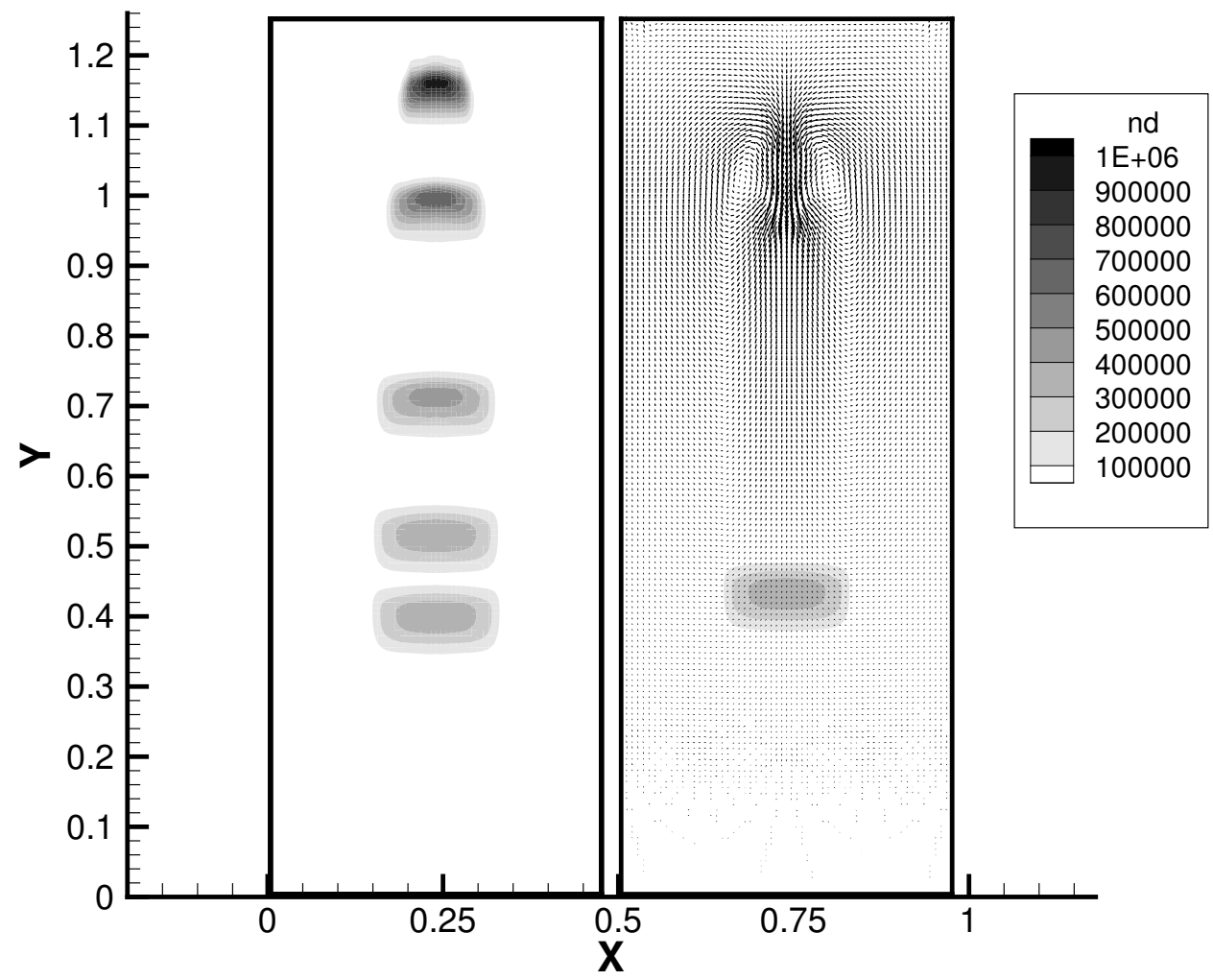

Figure 1: A schematic of the computational domain. Also shown is the simulated dispersion of a $0.2 \mathrm{~s}$ pulse of spherical solid particles $\left(d_{0}=6.25 \mathrm{~mm}\right.$, $\rho_{s}=1500 \mathrm{~kg} / \mathrm{m}^{3}$ ) dissolving over $t_{d}=4.22 \mathrm{~s}$. The liquid velocity field is also shown at time $t=3.0 \mathrm{~s}$. 


\subsection{Model development}

The domain geometry is two dimensional rectangular with a centrally located inlet, and pressure boundaries on the top wall to allow displaced fluid to exit (see Figure 1). The penetration depth achieved is described by the vertical centroid position (weighted by particle number density) at specific times.

Numerical simulation is undertaken using the two-fluid Eulerian-Eulerian approach, where both the solid and liquid phases are modelled as continuous, interpenetrating, and incompressible media. The relevant transport equations are those for multiphase flow with mass transfer. The continuity equations for dissolution take the form:

\section{Liquid Phase}

$$
\frac{\partial}{\partial t}\left(\rho_{l} \alpha_{l}\right)+\nabla \cdot\left(\alpha_{l} \rho_{l} \mathbf{U}_{l}\right)=\dot{m}_{l s} ;
$$

\section{Solid Phase}

$$
\frac{\partial}{\partial t}\left(\rho_{s} \alpha_{s}\right)+\nabla \cdot\left(\alpha_{s} \rho_{s} \mathbf{U}_{s}\right)=-\dot{m}_{l s} ;
$$

where $\dot{m}_{l s}$ represents the mass flow rate from the solid phase into the liquid phase per unit volume. The momentum equations are:

\section{Liquid Phase}

$$
\begin{aligned}
& \frac{\partial}{\partial t}\left(\alpha_{l} \rho_{l} \mathbf{U}_{l}\right)+\nabla \cdot\left(\alpha_{l} \rho_{l} \mathbf{U}_{l} \mathbf{U}_{l}\right) \\
= & \nabla \cdot\left(\alpha_{l} \boldsymbol{\tau}_{l}\right)-\alpha_{l} \nabla P+\alpha_{l} \rho_{l} \mathbf{g}-\mathbf{F}_{\mathbf{S}}^{(\mathbf{D})}-\mathbf{F}_{\mathbf{S}}^{(\mathbf{M T})}-\mathbf{F}_{\mathbf{S}}^{(\mathbf{L})}-\mathbf{F}_{\mathbf{S}}^{(\mathbf{A M})}
\end{aligned}
$$

\section{Solid Phase}

$$
\begin{aligned}
& \frac{\partial}{\partial t}\left(\alpha_{s} \rho_{s} \mathbf{U}_{s}\right)+\nabla \cdot\left(\alpha_{s} \rho_{s} \mathbf{U}_{s} \mathbf{U}_{s}\right) \\
= & -\alpha_{s} \nabla P+\alpha_{s} \rho_{s} \mathbf{g}+\mathbf{F}_{\mathbf{S}}^{(\mathbf{D})}+\mathbf{F}_{\mathbf{S}}^{(\mathbf{M T})}+\mathbf{F}_{\mathbf{S}}^{(\mathbf{L})}+\mathbf{F}_{\mathbf{S}}^{(\mathbf{A M})}+\mathbf{F}_{\mathbf{S}}^{(\mathbf{S P})} .
\end{aligned}
$$


Here the subscripts ' $s$ ' and ' $l$ ' refer to the solid and liquid phases respectively and $\boldsymbol{\tau}_{l}$ is the liquid viscous stress term. Variables $P, \rho$ and $\mathbf{g}$, represent the pressure, density and gravity, whereas $\alpha$ refers to the volume fraction. The terms $\mathbf{F}_{\mathbf{S}}^{(\mathbf{D})}, \mathbf{F}_{\mathbf{S}}^{(\mathbf{M T})}, \mathbf{F}_{\mathbf{S}}^{(\mathbf{L})}$ and $\mathbf{F}_{\mathbf{S}}^{(\mathbf{A M})}$ denote interfacial momentum transfer due to drag, mass transfer, lift and added mass, respectively. In the solid phase an additional term $\mathbf{F}_{\mathbf{S}}^{(\mathbf{S P})}$ denoting the effect of solids pressure in addition to the equilibrium pressure $P$ is included to allow for particle-particle interactions $[10,11]$. This prevents particle volume fractions from rising to unrealistic values (See Holbeach et al. [9] for further discussion of the various terms). A second order upwind advection differencing scheme, with Van-Leer flux limiting [12] is used for all transported variables as this helps reduce numerical smearing at the phase boundaries. Grid independence is achieved via a slightly non uniform $(61 \times 157)$ grid, with an approximate grid spacing of $0.8 \mathrm{~mm}$. Time step independence is achieved using fixed time stepping of $2 \mathrm{~ms}$, while convergence is considered when all normalised residuals are less than $2 \times 10^{-5}$.

\subsection{Dissolution}

The particles are assumed to dissolve via a simple dissolution model, whereby their diameter $d$ decreases linearly with time according to equation (5) (See earlier work by Holbeach et al. [9] for details).

$$
\begin{aligned}
& d(t)=d_{0}\left(1-\frac{t}{t_{d}}\right) \text { for } t \leq t_{d}, \\
& \text { where } \quad t_{d}=\frac{\rho_{s} d_{0}}{k_{m} \Delta C} .
\end{aligned}
$$

Here $d_{0}, k_{m}$ and $\Delta C$ refer to the initial particle diameter, mass transfer coefficient, and concentration difference between the particle interface and the fluid bulk, respectively. The parameter $t_{d}$ is the dissolution time (that is, the time it takes the particle to dissolve completely). The parameters $\Delta C$ 
and $k_{m}$ are assumed to be constant. While the concentration difference is unlikely to change significantly in dilute systems, $k_{m}$ will depend upon both the particle diameter and slip velocity. However, for the bulk of the particles dissolution history, Langberg and Nilmani [6] shows it to be relatively constant for diameter.

By considering a short pulse (relative to the particulate dissolution time), assumptions may be made regarding the dissolution of all particles in the system.

- The pulse is short enough $\left(t_{p}=0.2 \mathrm{~s}\right)$ that all particles may be regarded as having been exposed to the liquid for the same length of time. This allows us to ignore the fact that solids entering first start dissolving before those which enter later. In the simulation we assume that dissolution begins once all the particles have entered.

- All particles experience the same (constant) slip velocity, in regard to dissolution. This assumption allows us to assume that all particles have the same (constant) mass transfer coefficient, allowing equation (5) to be used for all particles. Note that the constant slip velocity assumption only applies to the formulation of equation (5). Slip velocities actually calculated within the two-fluid calculations will vary in time and position, in general. However, this assumption is reasonable as all particles fall at approximately the same rate through what can be regarded as an essentially stationary liquid.

- Lastly, we assume that the influence of the dissolution process upon the physical properties of the liquid phase, is negligible.

Given the above assumptions, the history of individual particles is then irrelevant and all particles may be considered to dissolve at exactly the same rate, and hence have the same radius at any time, given by equation (5). Momentum, mass transfer and transient drag considerations associated with the dissolution process, are discussed in earlier work by Holbeach et al. [9]. 


\section{Discussion}

Figure 1 shows the typical evolution of particles dissolving and dispersing after entering the domain. The particles spread horizontally and vertically as they descend through the domain; however, their vertical settling velocity is slowed by the inclusion of dissolution. This reduction in setting velocity during the plume's evolution is due to the changing instantaneous terminal velocity as the particles reduce in size. The velocity field is also shown in Figure 1. The fluid within the domain is initially stationary. The particles impart some momentum to the liquid phase upon entering, setting up a liquid velocity field, which in turn affects the particles' motion. Such simulations are discussed in more detail in Holbeach et al. [9].

The final depth achieved by dissolving spherical particles in the pulse, was found to relate primarily to the instantaneous terminal velocity $U_{t}$ of the individual particles, their initial diameter $d_{0}$, and the rate of dissolution $t_{d}$. Note that the terminal velocity of the particles is itself a function of the solid and liquid densities, $\rho_{s}$ and $\rho_{l}$, the particle size $d(t)$, and liquid viscosity. As the particles dissolve, their radius reduces which changes their "instantaneous" terminal velocity.

For the special case of single small particle of diameter $d$ the terminal velocity is

$$
\left.U_{t}=\frac{\Delta \rho g d^{2}}{18 \mu_{l}} \quad \text { (Stokes flow }\right)
$$

Since particle relaxation time is very small for tiny particles, integrating equation (7) will give the final depth $\left(y_{\max }\right)$ achieved for particles dissolving according to equation 5 to be

$$
\Upsilon=\frac{1}{3} J
$$

where $\Upsilon$ is the dimensionless penetration depth $y_{\max } / d_{0}$, and $J$ is the dimensionless group, $\left[U_{t_{0}}\left(t_{d} / d_{0}\right)\right]$ which is ratio of the initial particle terminal 
velocity $U_{t_{0}}$ to the dissolution velocity. Thus the depth achieved by isolated particles in Stokes flow is directly proportional to $J$. In view of this simple linear relationship for a single, small particle, it is of interest to consider the relationship between $J$ and $\Upsilon$ for a pulse of large particles. In the case of swarms of multiple larger particles, additional effects such as hindered settling and finite particle relaxation time, become significant.

Plots of $\Upsilon$ versus $J$ derived from the simulation were produced at various stages of dissolution (see Figure 2). However, as the particles are not in Stokes flow initially, we use the following (empirical) relationship [14] to calculate the initial terminal velocity, from the terminal Reynolds number $\left(\operatorname{Re}_{t}=U_{t} \rho_{l} d / \mu_{l}\right)$

$$
\operatorname{Re}_{t}=\left(2.33 \mathrm{Ga}^{0.018}-1.53 \mathrm{Ga}^{-0.016}\right)^{13.3},
$$

where

$$
\mathrm{Ga}=\frac{d^{3} \rho_{l}\left(\rho_{s}-\rho_{l}\right) g}{\mu_{l}^{3}} .
$$

To achieve the same value for $J$, a denser dissolving particle must be given a shorter dissolution time due to its relatively higher terminal velocity. For example in our simulation involving particles of initial diameter $d_{0}=$ $6.25 \mathrm{~mm}$; a simulation with $t_{d}=3.0 \mathrm{~s}$ and $\rho_{s}=3000 \mathrm{~kg} / \mathrm{m}^{3}$, would have $J=$ 333.1 , since $U_{t}=0.694 \mathrm{~m} / \mathrm{s}$. If, however, $\rho_{s}=1010 \mathrm{~kg} / \mathrm{m}^{3}$, then $t_{d}=63.8 \mathrm{~s}$ to achieve $J=333.1$, then $U_{t}=0.032 \mathrm{~m} / \mathrm{s}$.

It was found (Figure 2) that similar penetration depths are achieved by dissolving solid particles with the same $J$ value, and the same fractional reduction in diameter. Individual points at the same $J$ value represent simulations with different density ratios (between $1.01<\rho_{s} / \rho_{l}<3.0$ ).

It appears that, as for single tiny particles, equation (8), $\log \Upsilon$ varies linearly with $\log J$, but with a slope different from 1 . However, the graph must be used with caution as it is derived for a specific value of $d_{0}(6.25 \mathrm{~mm})$ and a linear decrease in particle diameter over time. The relationship for 


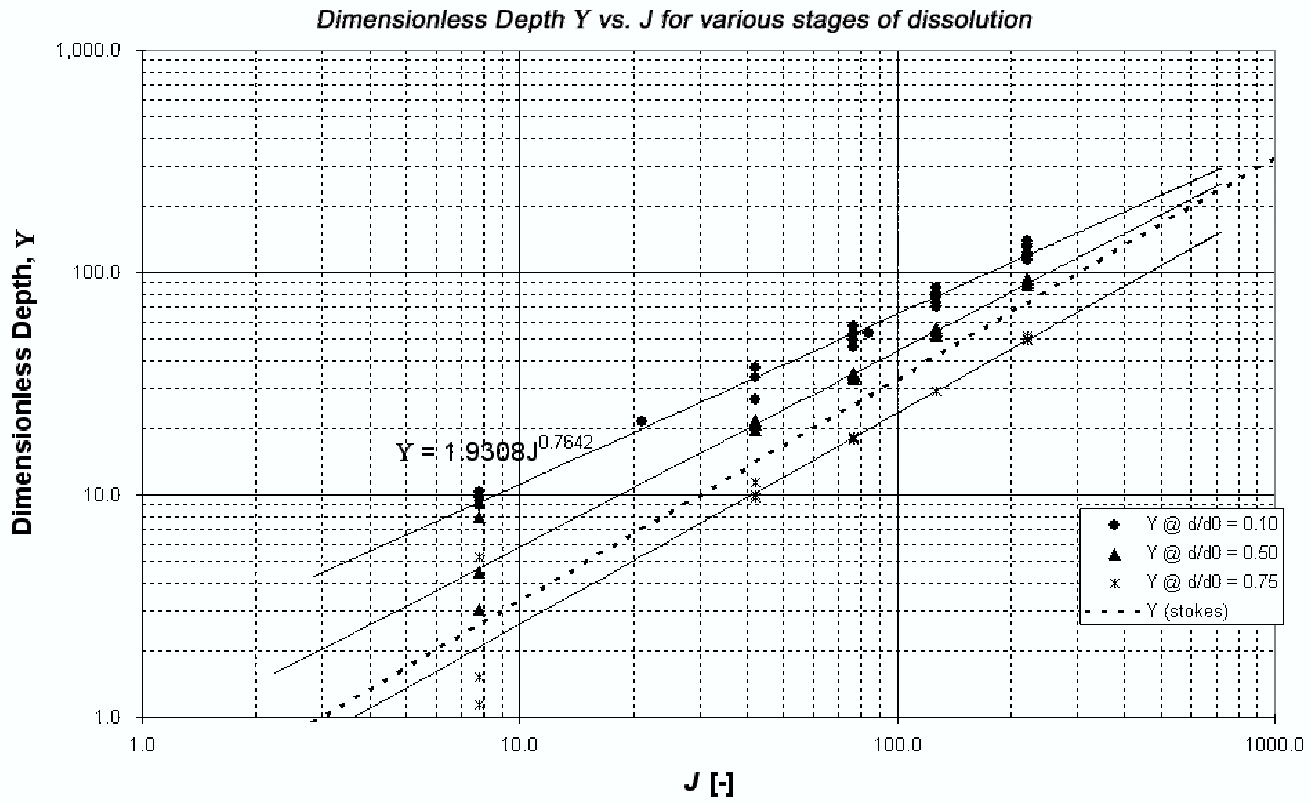

FiguRE 2: Dimensionless penetration depth is seen to increase with decreasing $J$. 
particles of other initial diameters, and for dissolution coupled to the flow field, will be the subject of further work.

This result implies that the final penetration depth is dependent primarily upon the initial conditions, and not on the dissolution history of the particles (for this special case of linear diameter change with time). Initial particle Reynolds number (based on the initial terminal velocity) is of the order of Re $\approx 1000$. In fact when $\rho_{s}=1100 \mathrm{~kg} / \mathrm{m}^{3}, d_{0}=6.25 \mathrm{~mm}, t_{d}=6 \mathrm{~s}$, the terminal Reynolds number only drops below 1 for the final $0.5 \mathrm{~s}$ of the dissolution. It is thus interesting to see that a relationship between $\Upsilon$ and $J$, similar to that for single particles in Stokes flow, exists. As expected, penetration depths achieved are significantly higher than would be predicted if the particles were in the Stokes regime, for a given $J$.

Settling velocities are higher than their instantaneous terminal velocity would predict in all simulations, except towards the very end of their dissolution (that is, when particles are smallest). This discrepancy is largest for short dissolution times (small $J$ ), and dense solids. The shorter the dissolution time, the less time the particle has to relax to its relevant instantaneous terminal velocity. Similarly, for denser solids, their dissolution time is necessarily shorter for a given $J$ value because the initial terminal velocity is now larger. Thus we see that for a given $J$ denser solids penetrate further than less dense solids. Nevertheless, the penetration depths for a given $J$ are similar, even over the range $1010<\rho_{s}<3000$, especially for $J>20$.

For $J<20$ the dissolution time for the particles is so short that our assumption regarding the pulse length being short relative to the dissolution time is made invalid. This accounts for the discrepancy seen at large $d / d_{0}$, for $J<20$. 


\section{Conclusion}

A simple power law relationship was found between penetration depth and the ratio of initial particle terminal velocity to the dissolution velocity for a pulse of large dissolving particles, dropped into an initially quiescent liquid. The relationship is similar to a corresponding relationship for settling single tiny particles. The result allows prediction of the approximate penetration depth that will be achieved by a cloud of particles where the particle radius falls linearly with time during dissolution.

Acknowledgment: This work has been made possible with the help of a G. K. Williams Cooperative Research Center Studentship. Special thanks also to David Fletcher of the University of Sydney, who helped in the development of specific user routines for drag within CFX.

\section{References}

[1] J. W. Brown and R. L. Reddy 'Electric Arc Furnace Steelmaking with Sponge Iron', Ironmaking and Steelmaking, pp. 24-31, 1979. C605

[2] M. A. Gilbertson et al. 'Isothermal Coarse Mixing: Experimental and CFD Modelling', Third U.K. National Conf. Incorporating 1st European Conf. on Thermal Sciences, Birmingham, Vol. 1, pp. 547-556, 1992. C605

[3] L. Meyer and D. Kuhn 'The Interaction of Very Hot Particles Falling into Water', Proc. of the 2nd Int. Symp. on Two-Phase Flow Modelling and Experimentation, Pisa, Italy, pp. 897-906, 1999. C605 
[4] D. F. Fletcher and P. J. Witt 'Numerical Studies of Multiphase Mixing with Application to some Small-Scale Experiments', Nuclear Eng. and Des., Vol. 166, pp. 135-145, 1996. C605

[5] K. M. Smith, M. R. Davidson, and D. F. Fletcher 'Dispersion of a Solids stream falling into a Stationary Liquid', Int. Conf. CFD in Min. Met. Proc. And Power Gen, Melbourne, pp. 133-140, 1997. C605

[6] D. E. Langberg and M. Nilmani 'The production of Nickel-Zinc Alloys by Powder Injection', Met. Trans B, Vol. 27B, pp. 780-787, 1996. C606, C610

[7] D. Apelian, R. O'Malley, and C. Dremann 'Injection of Non-Buoyant Particles', Proc. SCANINJECT II,Mefos and Jernkontoret, Lulea Sweeden, pp.7:1-7:33, 1980. C606

[8] M. S. Hameed and F. Y. Batta 'Experimental and Mathematical Studies of Natural Convection for Solid Salt Dissolution in Water', Can. J. Chem. Eng., Vol. 73, pp. 924-930, 1995. C606

[9] J. W. Holbeach and M. R. Davidson Modelling the dispersion of dissolving spherical particles. Prog. CFD J., Accepted for publication, 2003. C606, C609, C610, C611

[10] M. Syamlal and D. Gidaspow. (1985) 'Hydrodynamics of Fluidization: Prediction of Wall to Bed Heat Transfer Coefficients', AIChE J, Vol. 31, No. 1, pp. 127-135. C609

[11] M. Syamlal and T. J. O'Brien. (1988) 'Simulation of Granular Layer Inversion in Liquid Fluidized Beds', Int. J. Multiphase Flow, Vol. 14, No. 4, pp. 473-481. C609

[12] B. van Leer (1974) 'Towards the Ultimate Conservative Difference Scheme. II Monotonicity and Conservation Combined in a Second-Order Scheme', J. Comp. Phys., Vol. 14, pp. 361-370. 
[13] K. M. Smith 'Dispersion of solids dropped as a continuous stream into a liquid', PhD Thesis, The University of Melbourne, Australia, 2000 C606

[14] J. M. Coulson and J. F. Richardson et al. Chemical Engineering. Volume 2(4th Ed.), Butterworth Heinemann, pp. 105-108, 1996. C612 